\title{
ON THE POSSIBLE DIFFERENCES IN THE BULK CHEMICAL COMPOSITION OF THE EARTH AND THE MOON FORMING IN THE CIRCUMTERRESTRIAL SWARM
}

\author{
E. L. RUSKOL \\ O. Yu. Schmidt Institute of Physics of the Earth, Academy of Sciences, Moscow, U.S.S.R.
}

\begin{abstract}
The model of the origin of the Moon in the circumterrestrial swarm during the active stage of the Earth's growth [1, 2] receives now a support (see e.g. [3]). In this model the feeding substance is the same for both bodies. However two different processes may be mentioned of chemical fractionation between the Earth and the Moon.
\end{abstract}

\section{The Partial Loss of Volatile Elements from the Circumterrestrial Swarm}

The active formation of the swarm should take place when the mass of the growing Earth reached nearly a half of its present value. In this period the typical velocities of mutual collisions of particles captured into the swarm, were of the order of 3-7 km $\mathrm{sec}^{-1}$ in the whole sphere of action of the Earth. In subsequent collisions inside the swarm the velocities decreased. The collisions with the high energy $\left(10^{10}-10^{11} \mathrm{erg} \mathrm{g}^{-1}\right.$ etc.) led to multiple breaking up and both complete and partial evaporations of the particles' material, with the subsequent condensation of vapours on the solid particles. Different atoms and molecules could then escape into space under the action of the solar wind. Predominantly escaped the volatile substances (the water, the elements with the low melting temperatures $\mathrm{Pb}, \mathrm{Bi}, \mathrm{Tl}$, etc.).

It can be shown that the preplanetary cloud of a mass equal to the mass of terrestrial planets was rather transparent in radial direction $(\tau \lesssim 1)$ at the Earth's distance from the Sun, if the distribution of radii of the preplanetary bodies followed the law $\mathrm{d} N(r) \sim r^{-n} \mathrm{~d} r$, where $n \leqslant 3.5$ and $r_{\text {max }} \approx 10^{8} \mathrm{~cm}$. Thus, on the periphery of the circumterrestrial swarm also a transparency may be expected. In this region every free atom of lead, with the cross-section $\approx 10^{-15} \mathrm{~cm}^{2}$, had a chance to collide with a highenergy proton and to be ejected from the swarm once in two months, if the intensity of the solar wind was $2 \times 10^{8}$ protons $\mathrm{cm}^{-2} \mathrm{sec}^{-1}$, as at the present time. The condensation of that atom on a solid particle at the optical depth $\tau \approx 1$, took a time comparable with the period of revolution around the Earth, that is several months on the periphery of the swarm. If the intensity of the solar wind in the past was much higher than at present time, then the ejection of volatile elements from the periphery of the swarm would be more effective than their condensation on solid particles. On the contrary, at the accumulation of the Earth itself the volatile substances do not dissipate. It can be expected that the volatiles were retained also in the inner, more dense and obscure part of the swarm, which presumably has fallen onto the Earth. This fractionation may account for the deficiency, described by E. Anders and others, of volatile elements in lunar basalts and the enrichment of the latter in refractory ones in 
comparison with the terrestrial basalts [4]. It can be assumed that the primitive Moon was considerably depleted in water in comparison with the Earth. This strengthens the arguments against early lunar hydrosphere and atmosphere which were put forward earlier in the assumption of equal primitive composition of the Earth and the Moon [6].

The explanation of the deficiency of volatiles given by E. Anders seems to us less plausible, because he suggests the coincidence in time of a very short period of condensation of volatile elements at the earliest stage of the solar nebula (first $10^{5}-10^{6} \mathrm{yr}$ ) with the terminating period of growth of the Earth and the Moon. We believe that the time-scale of their growth was of the order of $10^{8} \mathrm{yr}$ [7], but not much less. Ringwood [5] explaines the deficiency of volatiles in the Moon by the solar wind. However his model of the origin of the Moon from a primitive massive iron-silicate Earth's atmosphere needs much more mechanical foundations (too short a time-scale, unclear mechanics of the formation of a satellite from an atmosphere etc.).

\section{The Selection of the Finest Particles at Their Capture into the Circumterrestrial Swarm and Resulting Enrichment of the Primitive Lunar Matter in Silicates}

If the Earth's core consists from iron, the Earth should contain nearly $35 \%$ of iron by weight. Meanwhile, the Moon contains only $14 \%$ of iron $[10,11]$.

We are tempting to explain this difference by the peculiarities of the formation of the circumterrestrial swarm from the preplanetary cloud, if in that cloud either (a) separately existed the iron and the silicate particles which had systematic differences in size due to some differences in physico-mechanical properties; or (b) there were no pure iron or silicate particles but the iron content increased with the particles dimension because the iron-rich bodies had more chance to survive in collisions and to grow up.

As Orowan has pointed out [9], the silicate particles are brittle and give fine debris in collisions, meanwhile the iron particles are plastic-ductile even at low temperatures and can coalesce at collisions. Altshuler and Sharipdjanov [11] also discuss some reasons stimulating the advanced growth of the iron particles in the preplanetary cloud.

As we have shown [2, part III], in the process of capture of particles into the circumterrestrial swarm, their size distribution changes even in the case without the breaking-up. If to approximate these distributions by power functions in the form $\mathrm{d} N(r) \sim r^{-n} \mathrm{~d} r$, then the power index $n_{1}$ for the interplanetary particles transforms into $n_{2}$ immediately after the capture of them into the swarm according the formula:

$$
n_{2}=2 n_{1}-3
$$

In particular, the index $n_{1}=3.5$ found for the preplanetary bodies $[7,8]$ transforms into $n_{2}=4$, which signifies the predominant capture of the finest component of the interplanetary assembly of particles and the permanent enrichment of the swarm by this component. The fragmentation of captured particles strenthens this effect. This 
selection does not affect the size distribution in the whole Earth's zone, if the mass of the swarm is small relative to the mass of all matter in the zone. At the same time the growing Earth accretes all particles without choice.

In both cases (a) and (b) mentioned above the enrichment of the swarm by the finest component signifies also the enrichment in silicates relative to iron. The case (b) may be illustrated by a numerical example.

Suppose the increase in $\mathrm{Fe}$ : $\mathrm{Si}$ ratio in bodies from the smallest particles to the greatest preplanetary bodies corresponds to the change of their density in the form:

$$
\delta(r)=\delta\left(r_{\min }\right)\left[1+a \log \left(r / r_{\min }\right)\right] .
$$

The ratio $r_{\max } / r_{\min }$ can be taken equal $10^{12}-10^{13}$. Suppose that $\delta\left(r_{\min }\right)=2.5 \mathrm{~g} \mathrm{~cm}^{-3}$, roughly the density of the lightest silicates; and that $\delta\left(r_{\max }\right)=4.5 \mathrm{~g} \mathrm{~cm}^{-3}$, roughly the density of the matter of the Earth at zero pressure. Then the average density for the assembly of particles of the size distribution with $n_{1}=3.5$ (preplanetary matter) will be $\bar{\delta}_{1}=4.4 \mathrm{~g} \mathrm{~cm}^{-3}$, and for that with $n_{2}=4.0$ (the swarm matter) $\bar{\delta}_{2}=3.5 \mathrm{~g} \mathrm{~cm}^{-3}$. We see that the values of $\bar{\delta}_{1}$ and $\bar{\delta}_{2}$ only slightly differ from the average densities correspondingly of the Earth and of the Moon. Thus the model of the circumterrestrial swarm permits to explain the difference in $\mathrm{Fe}: \mathrm{Si}$ ratio in the bulk composition of the Earth and the Moon.

\section{References}

[1] Schmidt, O. Yu.: 1959, A Theory of the Origin of the Earth, Lawrence and Wishart, London.

[2] Ruskol, E. L.: 1960, Sov. Astron. AJ 4, 657 (I); ibid. 7, 221, 1963 (II); 1971, Astron. Zh. 48, 819 (III).

[3] Kaula, W. M.: 1971, Rev. Geophys. Space Phys. 9, 217-38.

[4] Ganapathy, R., Keays, R. R., Laul, I. C., and Anders, E.: 1970, Proc. Apollo 11 Lunar Sci. Conf. $2,1117$.

[5] Ringwood, A. E.: 1970, Earth Planetary Sci. Letters 8, 131.

[6] Safronov, V. S. and Ruskol, E. L.: 1962, Proc. XIII Internat. Astronaut. Congr., Varna, 42.

[7] Safronov, V. S.: 1969, The Evolution of the Preplanetary Cloud and the Formation of the Earth and Planets (in russian), Nauka, Moscow.

[8] Dohnany, I. S.: 1967, in J. L. Weinberg (ed.), 'Collisional Model of Meteoroids', Proc. Symp. Honolulu, Hawaii.

[9] Orowan, E.: 1969, Nature 222, 867.

[10] Reynolds, R. T. and Summers, A. L.: 1969, J. Geophys. Res. 74, 2494.

[11] Altschuler, L. V. and Sharipdjanov, I. I.: 1971, Izv. An S.S.S.R., Fiz. Zemlii, No. 4, 3. 